The following document is a non peer-reviewed pre-print published on EarthArXiv. 


\title{
Bayesian elastic Full-Waveform Inversion using Hamiltonian Monte CARLO
}

Preprint, compiled SePtember 9, 2019

\author{
Lars Gebraad $^{1 *}$, Christian Boehm ${ }^{1}$, and Andreas Fichtner ${ }^{1}$ \\ ${ }^{1}$ Institute of Geophysics, ETH Zürich, Zürich, Switzerland
}

\begin{abstract}
We present a proof of concept for Bayesian elastic full-waveform inversion in 2-D. This is based on (1) Hamiltonian Monte Carlo sampling of the posterior distribution, (2) the computation of misfit derivatives using adjoint techniques, and (3) a mass matrix tuning of the Hamiltonian Monte Carlo algorithm that accounts for the different sensitivities of seismic velocities and density.

We apply our method to two synthetic end-member scenarios with different dimension $D$ that are particularly relevant in the context of full-waveform inversion: low-dimensional models $(D<100)$ with potentially large variations in material parameters, and high-dimensional models $\left(D>30^{\prime} 000\right)$ describing smaller-scale variations of lower amplitude relative to some background.

For both end members, the Hamiltonian Monte Carlo sampling reliably recovers important aspects of the posterior, including means, covariances, skewness, as well as 1-D and 2-D marginals. Depending on the strength of material variations, the posterior can be significantly non-Gaussian. This suggests to replace local methods for uncertainty quantification based on Gaussian assumptions by proper sampling of the posterior. In addition to $\mathrm{P}$-wave and S-wave velocity, the sampling provides constraints on density structure that are free from subjective regularization artifacts.
\end{abstract}

\section{INTRODUCTION}

\subsection{Full-waveform inversion}

While having been conceptualized already in the late 1970's and early 1980's [1, 2, 3, 4, 5], practical full-waveform inversion (FWI) is a comparatively recent addition to the seismological toolbox. Based on numerical wave propagation through potentially complex Earth models, it is the natural extension of ray tomography $[6,7,8,9,10,11]$ and finite-frequency tomography $[12,13,14,15,16]$. In recent years, successful applications of FWI have been reported in seismic exploration [17, 18, 19], in regional studies [20, 21, 22, 23], and for the whole Earth [24, 25, 26].

In addition to the computational requirements of numerical wave propagation, the non-linearity of the inverse problem has been one of the major impediments in the application of FWI. Being closely related to the challenge of finding an adequate initial model, it was recognized early on that non-linearity may be tamed through the incorporation of sufficiently low frequencies $[5,27,28]$. Attempts to mitigate non-linearity caused by cycle skipping led to the development of numerous misfit functionals that are more suitable than the traditional $L_{2}$ waveform difference $[4,5]$, especially when the available frequency band is rather limited [29, 30, 31, 32, 33, 34].

Non-linearity can in principle be handled elegantly by Monte Carlo sampling [35, 36]. However, the high-dimensionality of the model space paired with the computational costs of the forward problem, have so far limited its applicability to lowdimensional special cases [37, 38, 39]. For the same reasons, resolution and uncertainty analysis in FWI is still mostly local, making the assumption of a Gaussian posterior centered near a hopefully meaningful approximation of the maximumlikelihood model [40, 41, 42, 43, 44].

With this work, we explore non-linearity and uncertainty quantification in FWI using a recently developed sampling method, known as Hamiltonian Monte Carlo (HMC) [45, 46, 47, 48, 49]. Exploiting derivative information, HMC may solve high-dimensional problems where widely-used variants of the Metropolis-Hastings algorithm [50] tend to fail.

\subsection{Objectives and outline}

Our primary objective is a proof of principle that HMC can be successfully applied to two end-member cases of 2-D elastic FWI: (1) the non-linear search for coarse and a priori poorly known models that may serve as plausible starting points for subsequent spatial refinements, and (2) the probabilistic inversion for smaller-scale variations within more limited bounds, set, for instance, by the previous coarse-scale inversion combined with geologic prior knowlege.

We begin, in section 2, with a summary of the necessary theoretical background, including Bayesian inference, Markov chain Monte Carlo, Hamiltonian Monte Carlo, and numerical seismic wave propagation. In section 3 , we consider comparatively low-dimensional FWI, with 75 free parameters in total. This corresponds to the end-member case (1) described above. Section 4 is focused on high-dimensional problems. Specifically, we show that a single HMC chain can provide uncertainty information for $>30^{\prime} 000$ material parameters locally, while the algorithm also allows us to globally explore a posterior for as many parameters. Finally, in section 5, we provide a detailed discussion of advantages and drawbacks of the method. Also, we indicate possible improvements that are likely to increase the efficiency of the 
sampler, possibly allowing it to address higher-dimensional 3-D problems in the future.

\section{TheORETICAL BACKGROUND}

We consider 2-D elastic, isotropic models with density, $\rho$, Swave velocity, $v_{s}$, and $\mathrm{P}$-wave velocity, $v_{p}$, as free parameters. The HMC algorithm can be readily applied to FWI, as gradients of the misfit function can be conveniently computed using adjoint techniques $[51,52,53,54,55,56]$. There are, however, important technical details concerning target models, algorithm tuning, and model priors that affect the efficiency of the sampling. The following subsections comprise a short introduction to the theory of basic HMC sampling, and the synthesis of FWI and HMC. For a complete theoretical overview of and possible extensions to HMC we refer to [45] and [46]. Summaries of FWI theory can be found in [57], [58] and [59].

\subsection{Bayesian inference and Markov Chain Monte Carlo $(M C M C)$}

To set the stage and to establish basic notation, we begin with a brief recapitulation of Bayesian inference [60, 61]. For this we define $\mathbf{m}$ as an $n$-dimensional vector containing values of the discretized material properties $\rho, v_{s}$ and $v_{p}$. Information on $\mathbf{m}$ available prior to the analysis of any data is decribed by the probability density function (PDF) $p(\mathbf{m})$. Similarly, the prior probability of observing data $\mathbf{d}_{\text {obs }}$ given a specific $\mathbf{m}$ is encoded by a conditional PDF $p\left(\mathbf{d}_{\mathrm{obs}} \mid \mathbf{m}\right)$, usually referred to as the likelihood function. Both priors, $p(\mathbf{m})$ and $p\left(\mathbf{d}_{\mathrm{obs}} \mid \mathbf{m}\right)$, can be combined into the posterior PDF $p\left(\mathbf{m} \mid \mathbf{d}_{\text {obs }}\right)$ using Bayes theorem,

$$
p\left(\mathbf{m} \mid \mathbf{d}_{\mathrm{obs}}\right)=\frac{p\left(\mathbf{d}_{\mathrm{obs}} \mid \mathbf{m}\right) p(\mathbf{m})}{p\left(\mathbf{d}_{\mathrm{obs}}\right)} .
$$

The evidence $p\left(\mathbf{d}_{\text {obs }}\right)=\int p\left(\mathbf{d}_{\text {obs }} \mid \mathbf{m}\right) p(\mathbf{m}) d \mathbf{m}$ normalizes the posterior $p\left(\mathbf{m} \mid \mathbf{d}_{\mathrm{obs}}\right)$, which contains all possibly available information on models $\mathbf{m}$ given observations $\mathbf{d}_{\text {obs }}$. The likelihood function is typically written as exponential of a misfit function $\chi\left(\mathbf{m}, \mathbf{d}_{\mathrm{obs}}\right)$,

$$
p\left(\mathbf{d}_{\mathrm{obs}} \mid \mathbf{m}\right)=e^{-\chi\left(\mathbf{m}, \mathbf{d}_{\mathrm{obs}}\right)} .
$$

The misfit function serves as a measure of fit between observed data $\mathbf{d}_{\text {obs }}$ and synthetic data $\mathbf{d}$ computed from $\mathbf{m}$ via the solution of the forward modelling equations.

The posterior $p\left(\mathbf{m} \mid \mathbf{d}_{\mathrm{obs}}\right)$ is an $n$-dimensional PDF that is usually not known explicitly. Therefore, quantities of interest, such as means, (co)variances or marginal PDFs, are typically approximated by Markov chain Monte Carlo (MCMC) sampling of $p\left(\mathbf{m} \mid \mathbf{d}_{\mathrm{obs}}\right)$. All MCMC methods suffer from the curse of dimensionality in some form. Widely used variants of the MetropolisHastings algorithm [62, 63, 50,35], for instance, require increasingly smaller step sizes as the dimension $n$ grows, in order to ensure reasonable acceptance rates of proposed models. As a consequence, model space exploration is slow, and subsequent samples are highly correlated. Hamiltonian Monte Carlo (HMC), outlined in the following paragraphs, has been designed to overcome this problem, and to enable long-distance moves through model space while maintaining high acceptance rates $[64,45,46]$.

\subsection{Hamiltonian Monte Carlo}

Originally developed for molecular dynamics under the name hybrid Monte Carlo [64], Hamiltonian Monte Carlo (HMC) is now commonly used for the subset of sampling problems where gradients of the posterior $p\left(\mathbf{m} \mid \mathbf{d}_{\mathrm{obs}}\right)$ with respect to the model parameters $\mathbf{m}$ are easy to compute. The cost of generating independent samples with HMC under increasing dimension $n$ grows as $O\left(n^{5 / 4}\right)$ [45], whereas it grows as $O\left(n^{2}\right)$ for standard Metropolis-Hastings [65].

HMC constructs a Markov chain over an $n$-dimensional probability density function $p(\mathbf{m})$ using classical Hamiltonian mechanics [66]. The algorithm regards the current state $\mathbf{m}$ of the Markov chain as the location of a physical particle in $n$-dimensional space $\mathbb{M}$. It moves under the influence of a potential energy, $U$, which is defined as

$$
U(\mathbf{m})=-\ln p(\mathbf{m}) .
$$

In the case of a Gaussian probability density $p$, the potential energy $U$ is equal to the least-squares misfit $\chi(\mathbf{m})$. To complete the physical system, the state of the Markov chain needs to be artificially augmented with momentum variables $\mathbf{p}$ for every dimension and a generalized mass for every dimension pair. The collection of resulting masses are contained in a positive definite mass matrix $\mathbf{M}$ of dimension $n \times n$. The momenta and the mass matrix define the kinetic energy of a model as

$$
K(\mathbf{p})=\frac{1}{2} \mathbf{p}^{T} \mathbf{M}^{-1} \mathbf{p} .
$$

In the HMC algorithm, the momenta $\mathbf{p}$ are drawn randomly from a multivariate Gaussian with covariance matrix $\mathbf{M}$. The location-dependent potential and kinetic energies constitute the total energy or Hamiltonian of the system,

$$
H(\mathbf{m}, \mathbf{p})=U(\mathbf{m})+K(\mathbf{p}) .
$$

Hamilton's equations

$$
\frac{d \mathbf{m}}{d \tau}=\frac{\partial H}{\partial \mathbf{p}}, \quad \frac{d \mathbf{p}}{d \tau}=-\frac{\partial H}{\partial \mathbf{m}} .
$$

determine the position of the particle as a function of the artificial time variable $\tau$. We can simplify Hamilton's equations using the fact that kinetic and potential energy depend only on momentum and location, respectively,

$$
\frac{d \mathbf{m}}{d \tau}=\mathbf{M}^{-1} \mathbf{p}, \quad \frac{d \mathbf{p}}{d \tau}=-\frac{\partial U}{\partial \mathbf{m}} .
$$

Evolving $\mathbf{m}$ over time $\tau$ generates another possible state of the system with new position $\tilde{\mathbf{m}}$, momentum $\tilde{\mathbf{p}}$, potential energy $\tilde{U}$, and kinetic energy $\tilde{K}$. Due to the conservation of energy, the Hamiltonian is equal in both states. Successively drawing random momenta and evolving the system generates a distribution of the possible states of the system. Thereby, HMC samples the joint momentum and model space, referred to as phase space. As we are not interested in the momentum component of phase space, we marginalize over the momenta by simply dropping them. This results in samples drawn from $p(\mathbf{m})$.

If one could solve Hamilton's equations exactly, every proposed state would be a valid sample of $p(\mathbf{m})$. Since Hamilton's equations for non-linear forward models cannot be solved analytically, the system must be integrated numerically. Suitable integrators are symplectic, meaning that time reversibility, phase 
space partitioning and volume preservation are satisfied [45, 67]. However, the Hamiltonian is generally not preserved exactly when explicit time-stepping schemes are used. In this work, we employ the leapfrog method as described in [45]. As the Hamiltonian is not preserved, the time evolution generates samples not exactly proportional to the original distribution. A Metropolis-Hastings correction step is therefore applied at the end of numerical integration.

In summary, samples are generated starting from a random model $\mathbf{m}$ in the following way:

1. Propose momenta $\mathbf{p}$ according to the Gaussian with mean $\mathbf{0}$ and covariance $\mathbf{M}$;

2. Compute the Hamiltonian $H$ of model $\mathbf{m}$ with momenta p

3. Propagate $\mathbf{m}$ and $\mathbf{p}$ for some time $\tau$ to $\tilde{\mathbf{m}}$ and $\tilde{\mathbf{p}}$, using the discretized version of Hamilton's equations and a suitable numerical integrator;

4. Compute the Hamiltonian $\tilde{H}$ of model $\tilde{\mathbf{m}}$ with momenta $\tilde{\mathbf{p}}$

5. Accept the proposed move $\mathbf{m} \rightarrow \tilde{\mathbf{m}}$ with probability

$$
p_{\text {accept }}=\min (1, \exp (H-\tilde{H})) \text {. }
$$

6. If accepted, use (and count) $\tilde{\mathbf{m}}$ as the new state. Otherwise, keep (and count) the previous state. Then return to 1 .

The main factor influencing the acceptance rate of the algorithm is the conservation of energy, $H$, along the trajectory. If the leapfrog integration has too large time steps, or the gradients of the misfit function are computed incorrectly (e.g., by badly discretizing the forward model), $H$ is less well conserved, and the algorithm's acceptance rate decreases.

The main cost of HMC, compared to other MCMC samplers, is the computation of the gradient $\partial U / \partial \mathbf{m}$ at every step in the leapfrog propagation. When gradients can be computed easily, HMC can provide improved performance for two reasons: (1) the reduced cost of generating independent samples, that is, the avoidance of random-walk behaviour [45], and (2) the better scaling of HMC with increasing dimension [65, 45].

The tuning parameters in HMC are simulation time $\tau$ and the mass matrix M. HMC has the potential to inject additional knowledge about the distribution $p$ via the mass matrix in order to enhance convergence significantly. At the same time, the abundance of tuning parameters also creates potential for choosing inefficient settings, leading to sub-optimal convergence. [48] and [67] both illustrate how to create relevant mass matrices for tomographic inverse problems.

We adapt the specific tuning strategy for the mass matrix in this study depending on the target, as illustrated in the following sections. However, for all targets we choose the size of the discrete time steps empirically such that the acceptance rate is close to the optimum of $65 \%$ [45]. This typically results in needing approximately 10 leap-frog steps per proposal, i.e. requiring this many forward and adjoint solves per proposal.

\subsection{Numerical seismic wave propagation}

Our inversions target 2-D vertical cross sections of isotropic wave velocities and density. For this, we consider the P-SV wave system, written in velocity-stress formulation as

$$
\begin{aligned}
\partial_{t} v_{x} & =\rho^{-1}\left(\partial_{x} \tau_{x x}+\partial_{z} \tau_{x z}\right) \\
\partial_{t} v_{z} & =\rho^{-1}\left(\partial_{x} \tau_{x z}+\partial_{z} \tau_{z z}\right) \\
\partial_{t} \tau_{x x} & =(\lambda+2 \mu) \partial_{x} v_{x}+\lambda \partial_{z} v_{z} \\
\partial_{t} \tau_{z z} & =(\lambda+2 \mu) \partial_{z} v_{z}+\lambda \partial_{x} v_{x}, \\
\partial_{t} \tau_{x z} & =\mu\left(\partial_{z} v_{x}+\partial_{x} v_{z}\right) .
\end{aligned}
$$

with the velocity vector $\left(v_{x}, v_{z}\right)$, the stress tensor components $\tau_{x x}, \tau_{z z}$ and $\tau_{x z}$, the Lamé coefficients $\lambda$ and $\mu$, and density $\rho$. All quantities are a function of position $\mathbf{x}=(x, z)$.

We discretize these differential equations using the fourth-order variant of the staggered-grid finite-difference scheme developed by [68]. As free parameters we use the P-wave velocity $v_{p}=$ $\sqrt{(\lambda+2 \mu) / \rho}$, the S-wave velocity $v_{s}=\sqrt{\mu / \rho}$, and density, $\rho$.

For the computation of sensitivity kernels, we use the adjoint method [51, 52, 53, 54, 55, 56]. Subsequently, we project the kernels onto the basis functions, used to represent the elastic medium. This yields the gradient needed in the HMC algorithm.

\subsection{Waveform misfit and tempering}

An important choice in the solution of an inverse problem is the misfit used to quantify differences between observed data $\mathbf{d}_{\text {obs }}$ and synthetic data $\mathbf{d}(\mathbf{m})$. It determines, among other things, the extent to which different parameters can be resolved. In the interest of simplicity, we choose the $L_{2}$ waveform difference,

$$
\chi_{L_{2}}(\mathbf{m})=\frac{1}{2} \sum_{\mathrm{i}}\left(\frac{d_{i, \mathrm{obs}}-d_{i}(\mathbf{m})}{\sigma_{i}}\right)^{2},
$$

where the indices $i$ denote time samples. The scalars $\sigma_{i}$ are the standard deviations per data point, corresponding to a diagonal data covariance matrix. More complex and in real-data applications more meaningful data covariances can be used; but this is beyond the scope of this synthetic study.

The data variances $\sigma_{i}^{2}$ can be parameters of the inversion, which may be estimated by hyperparameter inversion [69]. They should, however, not be changed to make the HMC sampler behave in a specific way [70]. Choosing an identical variance $\sigma_{i}^{2}=\sigma^{2}$ for all data points, makes $\sigma^{2}$ behave analogously to the temperature parameter $T$ in tempering [71, 72]. A tempered distribution $p_{T}$ is constructed from the original distribution $p$ as

$$
p_{\mathrm{T}}(\mathbf{m})=p\left(\mathbf{m} \mid \mathbf{d}_{\text {obs }}\right)^{1 / T} \propto \exp \left(-\frac{\chi}{T}\right)
$$

The variable $T$ determines the temperature of the tempered distribution $p_{T}$. We analyse the impact of changing temperature as a proxy for changing data variance (i.e., noise levels) in section 4.

Though the $L_{2}$ waveform difference (14) has been used traditionally in FWI studies [2, 4, 5, 73], other, and in practice often more suitable, misfits may be used [29, 30, 31, 32, 34].

HMC, as most other Monte Carlo samplers, does not necessarily require a globally convex misfit function. However, the absence 
of local minima generally improves convergence, sometimes at the expense of reduced resolution. For an illustration of the behaviour of HMC in multimodal posteriors and possible mitigations, we refer to [45]. The $L_{2}$ waveform misfit (14) is dominated by large-amplitude $\mathrm{S}$-waves, whereas lower-amplitude $\mathrm{P}$-waves have a smaller influence. This property will be reflected in the inversion results.

\subsection{Prior information}

All model priors used in this work are uniform distributions within certain bounds. The width of the prior reflects two endmember scenarios and objectives of Bayesian FWI: (1) To find a range of admissible initial models for deterministic FWI, a small number of model parameters will be used with a weak prior, that is, a broad uniform distribution. This mode of operation is related to global optimization in the potential presence of multiple local minima. (2) In contrast, a large number of model parameters with deviations from a well-known background is needed to constrain small-scale deviations. Their priors will be stronger, that is, the uniform distribution will be comparatively narrow. As with the misfit and data covariance model, the uniform prior was chosen for its simplicity. In practice, more complex and meaningful priors can be used, which for example introduce correlations (i.e. smoothness constraints on admissible models).

In the HMC sampling, any model with non-zero prior likelihood could be proposed. Consequently, the finite-difference simulations must be numerically stable for any model admitted by the prior. This requires a conservative choice of temporal and spatial sampling for the finite-difference simulations, dependent on the width (bounds) of the prior. In this work, all extreme values of the priors still result in numerically stable wavefield simulations.

\section{LOW-DIMENSIONAL MODEL SPACE SAMPLING}

We start with the case of a low-dimensional model space where Earth structure is a priori poorly known and represented by few basis functions. This scenario is intended to mimic the situation where plausible but yet simple initial models for a deterministic FWI need to be found, often in the presence of limited data (in spatial coverage, bandwidth, or both). The priors used are relatively wide with respect to the next section, with uniform distributions in the interval $2000 \pm 1000 \mathrm{~m} / \mathrm{s}$ for $v_{p}, 800 \pm 400$ $\mathrm{m} / \mathrm{s}$ for $v_{s}$, and $1500 \pm 500 \mathrm{~kg} / \mathrm{m}^{3}$ for $\rho$. The middle value is the background value to which the perturbations of each target are applied.

In the interest of simplicity and easy visualization, we consider two checkerboard patterns, shown in Fig. 1. Both checkerboard models are embedded in the same physical domain, and they share identical source-receiver setups. The domain is $125 \mathrm{~m}$ by $125 \mathrm{~m}$ wide, with absorbing boundaries at all sides except the top, where a free surface is implemented. Waves from two moment tensor sources are recorded by six receivers. The source time function is a Ricker wavelet with a central frequency of 50 $\mathrm{Hz}$. The number of checkerboard blocks is $5 \times 5$. Having three physical parameters, this corresponds to 75 free parameters.

The checkerboards, used to compute artificial data, differ in one aspect: The anomaly strength is either $10 \%$ or $25 \%$ relative to the background, and for all model parameters, $v_{p}, v_{s}$, and $\rho$. Variable anomaly strength allows us to investigate the effect of increasing non-linearity on algorithm performance and the posterior distributions. The weaker perturbations are referred to as checkerboard 1, whereas the stronger perturbations are referred to as checkerboard 2.

\subsection{Tuning strategy and starting models}

While the mass matrix $\mathbf{M}$ can in principle be any positive definite matrix, its design determines the effectiveness of HMC by controlling the relative speed of the particle (i.e., the model) for every separate dimension in model space. Ideally, all dimensions are explored equally fast. For linear inverse problems (with Gaussian prior and Gaussian noise model), this can be achieved with a mass matrix that equals the inverse posterior covariance [48].

The posterior covariance matrix is, by definition, not known a priori, and our inverse problem is not linear. Therefore, we employ a trial-and-error tuning strategy based on the acceptance rate and trace plots of preliminary runs of the Markov chain. For this, we first simplify the mass matrix (with dimensions $75 \times 75$ ) to three tuning parameters, with one mass for each parameter set $\left(m_{v_{p}}, m_{v_{s}}\right.$, and $\left.m_{\rho}\right)$,

$$
\mathbf{M}=\left[\begin{array}{lll}
m_{v_{p}} \mathbf{I}_{25} & & \\
& m_{v_{s}} \mathbf{I}_{25} & \\
& & m_{\rho} \mathbf{I}_{25}
\end{array}\right],
$$

where $\mathbf{I}_{25}$ stands for the $25 \times 25$ identity mass matrix. An added benefit is that the mass matrix is diagonal, which greatly accelerates the computation of kinetic energy (4) and the proposal of momenta.

The tuning of $m_{v_{p}}, m_{v_{s}}$ and $m_{\rho}$ is illustrated in Fig. 2. We start with a mass matrix equal to the identity matrix. During the first few hundred samples, the values of $v_{s}$ stabilize into two groups, as expected for the checkerboard. However, the values of $v_{p}$ and $\rho$ hardly move, suggesting that their masses are too large. Therefore, after around 2900 samples, we decrease $m_{v_{p}}$ and $m_{\rho}$. This leads to larger movement of the respective parameters; and after around 4000 samples also the values of $\rho$ have stabilized. To further increase the movement of $v_{p}$, we again decrease $m_{v_{p}}$. This sequence can be repeated several times. In this specific example, 3 iterations were sufficient to obtain reasonable values for $m_{v_{p}}, m_{v_{s}}$ and $m_{\rho}$.

The initial model for all inversions is chosen to be homogeneous. After the preliminary tuning chain, the samples after the last mass matrix update are used to supply initial models for subsequent Markov chains. These models are chosen at random.

\subsection{Sampling strategy and performance}

We sample the posterior by naïve parallelization on a computing cluster, meaning that we simultaneously run 20 to 40 chains with identical settings but different starting models. Autocorrelations of all parameters fall below 0.1 within $5 \%$ of the total chain length, and subsequently oscillate around zero. We terminate the chains empirically; when no apparent change to means and variances is observed over many samples the chain is assumed to be converged and is subsequently terminated. A detailed discussion about convergence can be found in section 5 . The results 

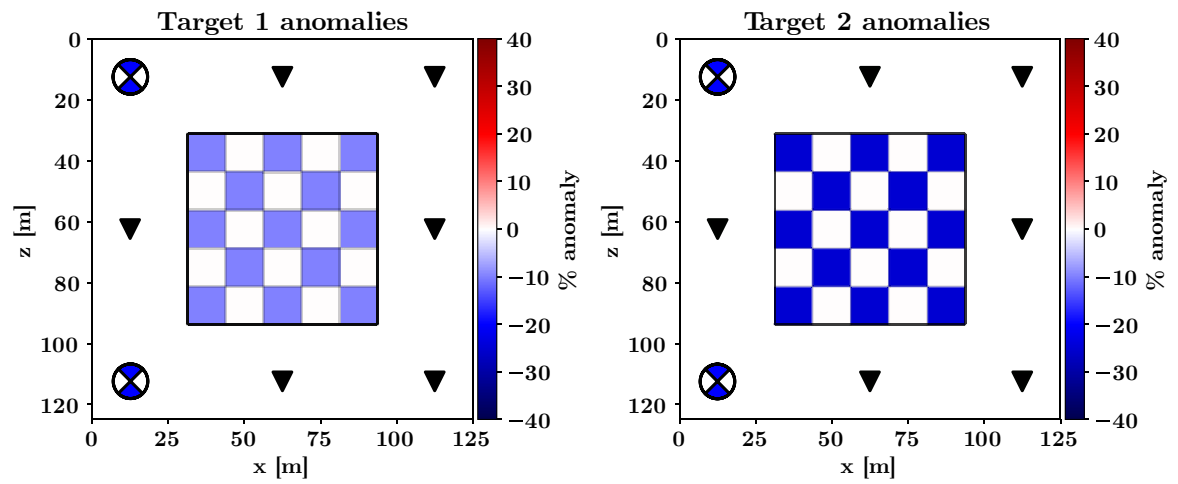

Figure 1: Checkerboard patterns in $v_{p}, v_{s}$, and $\rho$. The source-receiver setup and the domain dimensions are identical, with receivers ( $\mathbf{v})$ present both at depth and at the surface. The source mechanisms, represented by beachballs, are equally oriented.

are Markov chains with 100'000 samples (target 1) and 600'000 samples (target 2), respectively. Their properties are summarized in Table 1. The following subsections highlight interesting results from selected Markov chains. Posterior statistics for all targets are accessible in the electronic supplement.

\subsection{Marginal moments and maximum poster probability}

Though non-linearity implies non-Gaussianity, we begin the charactrization of the posterior with an analysis of means and standard deviations, shown in Figs. 3a,b for target 2. While the posterior mean $v_{s}$ model is nearly identical to the target $v_{s}$ model, larger differences between mean and target are visible for $v_{p}$ and $\rho$. This is also reflected in the standard deviations, which are significantly larger for $\rho$ and $v_{p}$ than for $v_{s}$. In this, somewhat limited, sense, $v_{s}$ is better resolved than $\rho$ and $v_{p}$. These results are plausible given the relative insensitivity of seismic waveforms to density, and the dominance of largeramplitude $\mathrm{S}$ waves over lower-amplitude $\mathrm{P}$ waves in the $L_{2}$ waveform misfit [74]. For all parameters, the magnitude of the standard deviations is much smaller than the uniform prior standard deviations $\left(\sigma_{\text {prior }}=\right.$ width $\left./ \sqrt{12}\right)$, indicating that we were able to reduce dispersion of the marginals using the FWI experiment, i.e. we 'learned something'.

As can be seen in Fig. 3, standard deviations depend on the target parameter value, in addition to depending on location relative to sources and receivers. The dependence of posterior standard deviations on the target itself is a consequence of non-linearity and non-Gaussianity, which is more explicitly expressed by the third statistical moment, the skewness

$$
S=\frac{E\left[(X-\bar{X})^{3}\right]}{E\left[(X-\bar{X})^{2}\right]^{3 / 2}},
$$

where $X$ is a physical parameter (e.g., $v_{s}$ ), $E[$.] denotes the mean, and $\bar{X}=E[X]$. The skewness is a measure of dispersion where positive and negative deviations contribute in opposite magnitude, due to the third power. Thereby, skewness is a measure of lopsidedness or asymmetry of a distribution. Nonzero values indicate whether the distribution is leaning heavily to one side, i.e., having asymmetric tails. A (multivariate) normal distribution, corresponding to the posterior of a linear inverse problem, has zero skewness. For illustration, a skew-normal distribution with two different skewness values is shown in Fig. 4.
Note that the mean, median, and mode are all different. This in turn implies that plotting the mean model is not a sufficient characterization for highly skewed or non-normal distributions.

Skewness in target 2, shown in Fig. 3, is non-zero for many parameters, indicating a non-Gaussian posterior. This suggests that the use of a Hessian approximation for uncertainty quantification in full-waveform inversion may not be sufficient.

Additionally shown in Fig. 3 is the model (sample) with the smallest misfit evaluated during sampling, i.e., the maximum poster probability (MAP) model. Although occasionally interpreted as the solution, it is most likely not the absolute global minimum. In this case, the MAP model actually reflects the target worse than the means of the Markov chain, especially for P-wave velocity.

\subsection{Joint distributions and inter-parameter moments}

In addition to the marginal statistical moments, sampling also allows us to visualize marginal or conditional distributions. An example of a 2-D marginal for the checkerboard targets is shown in Fig. 5. The two parameters visualized are P-wave velocities in neighboring blocks centered at $(78 \mathrm{~m}, 62 \mathrm{~m})$ and $(78 \mathrm{~m}, 46 \mathrm{~m})$. As a consequence of both non-linearity and ill-posedness, the marginal is significantly non-Gaussian, and the parameters are strongly dependent, even for target 1 with the lower-amplitude perturbations. This, again, highlights that uncertainty analysis based on a Gaussian approximation may have limited meaning.

Posterior samples provide information on covariance, which allows us to compute correlations between model parameters in the posterior. The correlation matrix for target 2 is shown in Fig. 6. A more physical interpretation can be done by selecting one column/row from the correlation matrix and plotting the values in the corresponding basis functions. This has been done for parameter 8 of target 2 in Fig. 7. In the electronic supplement correlations between all parameters are available. Higher-order co-moments (e.g. co-skewness, co-kurtosis) are also available from the samples, but are more difficult to interpret.

\section{High-DIMENSIONAL MODEL SPACE SAMPLING}

Following the consideration of a relatively low-dimensional case with large prior uncertainties, we continue with a highdimensional model space that is more suitable for the represen- 


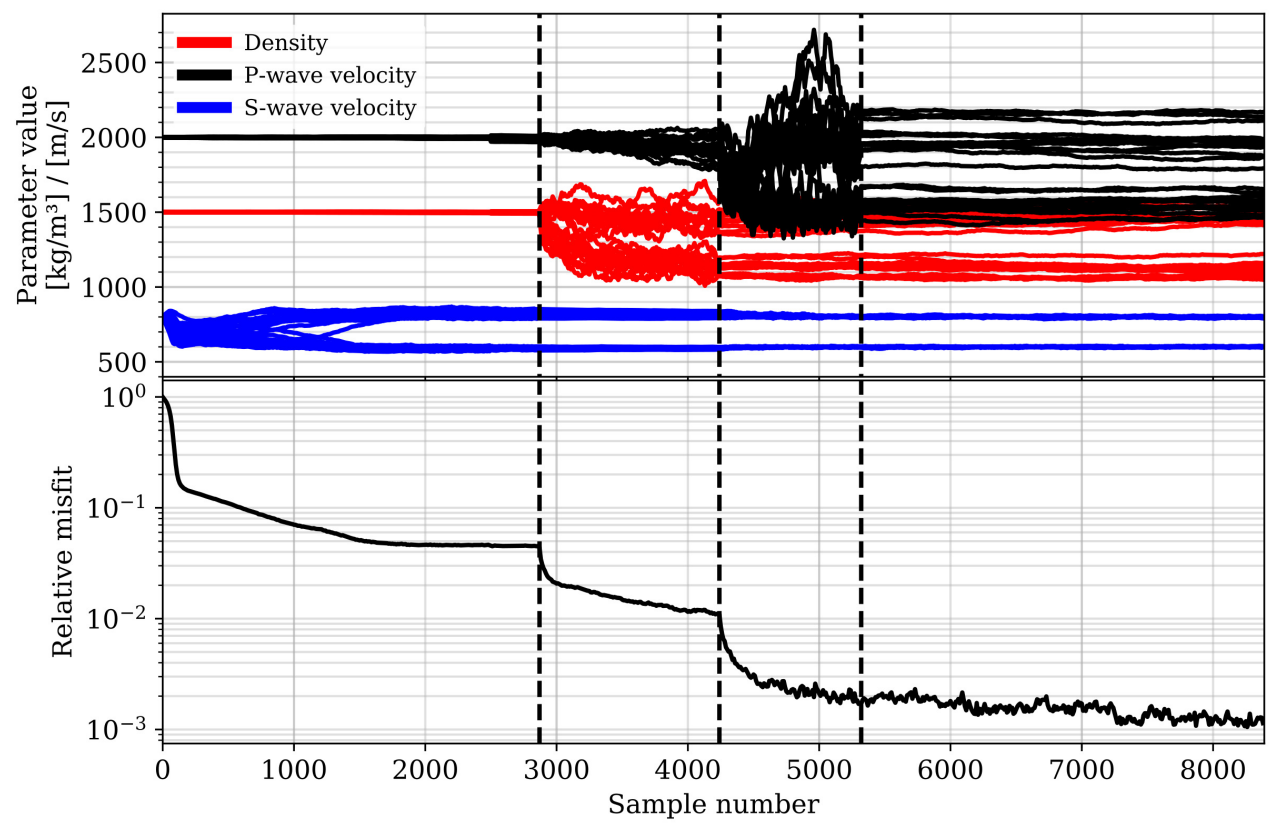

Figure 2: Iterative tuning of the mass matrix for checkerboard target 2 as given in Fig. 1. Top: Trace plot of an exploratory Markov chain. Each curve corresponds to one of the 75 model parameters. Coloring represents the parameter class. Bottom: The corresponding waveform misfit during this chain. Every vertical line represents an update of the three scalar masses. The parameters with the strongest impact on the misfit stabilize first.

tation of detailed geologic structures. Combined with lower prior uncertainties, this corresponds to a scenario where we seek smaller-scale variations relative to a coarse background model that is already well constrained.

Specifically, we construct a $32^{\prime} 400$-dimensional target that mimics a geological structure set in a transmission-dominated experiment. The free parameters are $v_{p}, v_{s}$ and $\rho$ defined on the $180 \times 60=10^{\prime} 800$ finite-difference grid points. The sources have random moment tensors and are positioned near the bottom of the domain. As source-time function we again use a Ricker wavelet with dominant frequency of $50 \mathrm{~Hz}$. Note that at the dominant frequency, the spatial structure is sub-wavelength. The structural target and source-receiver setup are shown in Fig. 8.

While the increased model space dimension acts to decelerate convergence relative to the low-dimensional checkerboard models, this is balanced by stronger prior knowledge, that is, smaller variations with respect to the background. The prior distributions are uniform in the interval $2000 \pm 100 \mathrm{~m} / \mathrm{s}$ for $v_{p}, 800 \pm 50$ $\mathrm{m} / \mathrm{s}$ for $v_{s}$, and $1500 \pm 100 \mathrm{~kg} / \mathrm{m}^{3}$ for $\rho$.

Additionally, we vary data variance to investigate the influence of the (assumed) noise level. The data variance is given by $\sigma^{2}$ in Eq. (14), and varied from $\sigma^{2}=10 \mu \mathrm{m}^{2}$ to $\sigma^{2}=1 \mu \mathrm{m}^{2}$ and finally to $\sigma^{2}=0.1 \mu \mathrm{m}^{2}$. We henceforth describe these values qualitatively as high $\left(10 \mu \mathrm{m}^{2}\right)$, medium $\left(1 \mu \mathrm{m}^{2}\right)$, and low $\left(0.1 \mu \mathrm{m}^{2}\right)$ data variance. The data noise described by it's variance was not added as synthetic noise to the data. As before, important characteristics of all chains are summarized in Table 1 and posterior statistics of all targets are accessible through the electronic supplement.

\subsection{Updated tuning strategy}

The tuning strategy from section 3.1, where the mass matrix is simplified to include only three parameters, works well when the data variance is high, i.e., when the typical set occupies a large model space volume. However, for lower data variances, the volume of the typical set shrinks quickly, and a more elaborate mass matrix tuning becomes necessary to ensure acceptable convergence.

Our strategy is based on the analysis of linear inverse problems, where the posterior covariance matrix can be shown to be the optimal mass matrix [67]. Since the posterior covariance is by definition a priori unknown, we choose an approximate approach. For this, we first run a shorter preliminary chain using the simple mass matrix tuning introduced in section 3.1. Based on these samples, we compute a rough estimate of the diagonal entries of the posterior covariance, which then serves as a more suitable mass matrix. Though this approach could in principle be repeated multiple times, we only use a single estimate from a previous chain.

Implicitly, this tuning strategy rests on the assumptions that the posterior is roughly Gaussian and that the postrior covariance matrix can be reasonably approximated by its diagonal estimated from a limited number of samples. The extent to which these assumptions hold, determines the effectiveness of the enhanced 

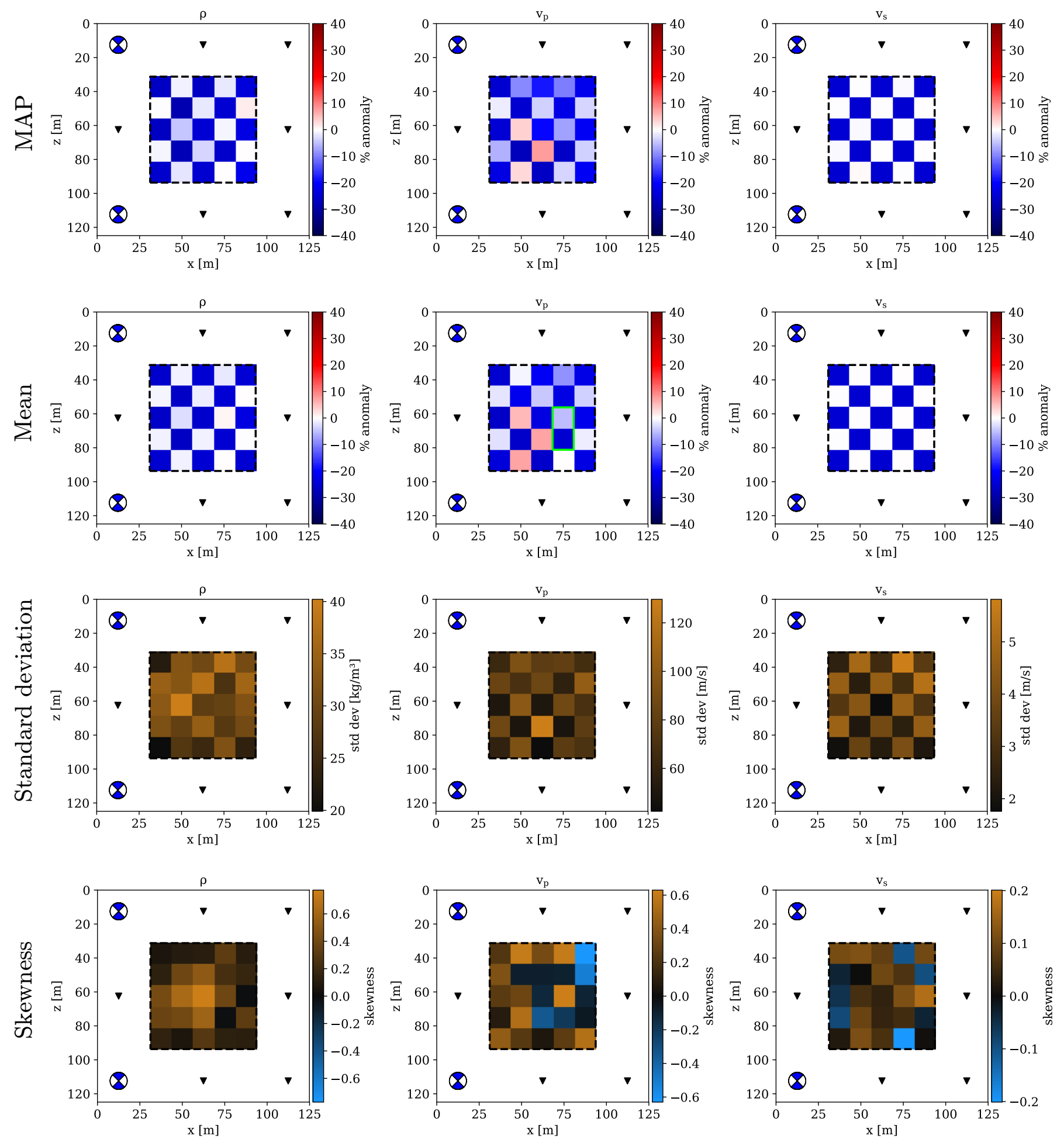

Figure 3: Summary of the posterior distribution, including the MAP point and first three statistical moments for checkerboard target 2 shown in Fig. 1. These quantities are computed from chain B in Table 1. While S-wave velocity in the MAP model is virtually indistinguishable from the respective means, density and P-wave velocity show deviations. Density and S-wave velocity are well resolved in the sense of having small standard deviations, but P-wave velocity is not as close to the true model for both the means as well as in the MAP model. As expected, the smallest standard deviations for all parameters occur close to the sources. A large portion of the parameters has non-zero skewness, indicating that these behave non-Gaussian. The green box plotted in the mean model refers to the parameters visualized in Fig. 5. 

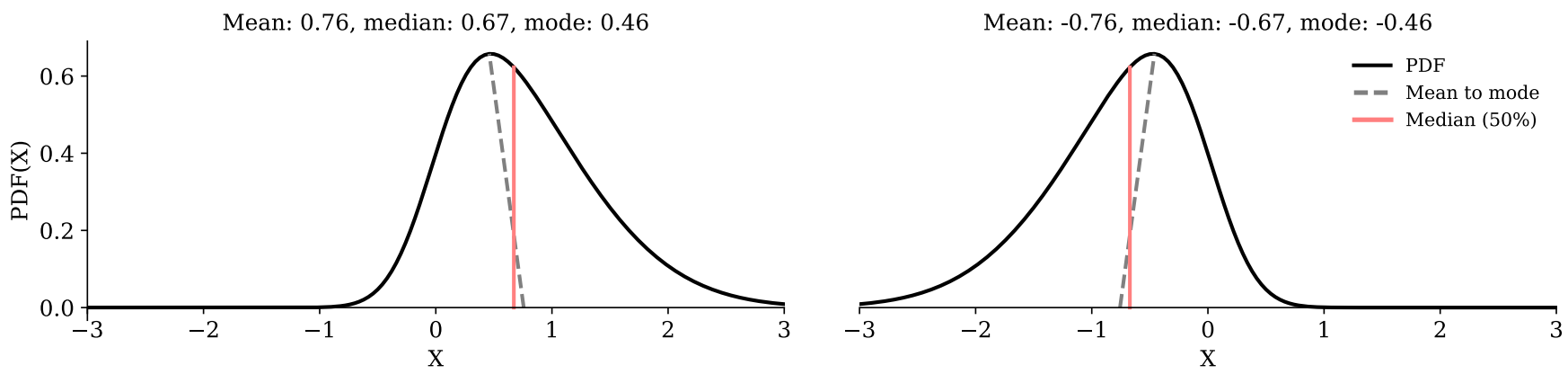

Figure 4: Two skew-normal distributions with positive (on the left hand side) and negative (on the right hand side) skew. The two lines plotted are: a line connecting the mean on the $\mathrm{x}$-axis with the mode (maximum of the distribution) and a line dividing the PDF area in half (median). The skew-normal distributions are described with location 0 , scale 1 , and skewness 5 (left) or -5 (right).
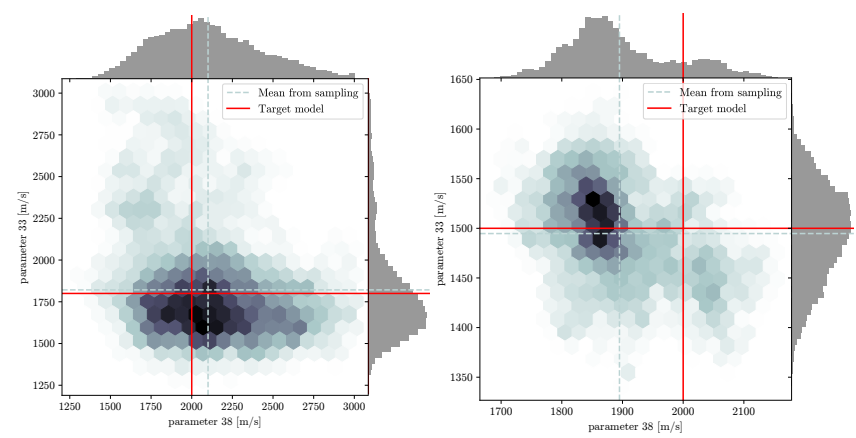

Figure 5: Examples of 2-D joint distributions for two highly skewed and correlated parameters of checkerboard targets 1 (left) and 2 (right), shown in Fig. 1. The two parameters are $\mathrm{P}$-wave velocity of adjacent basis functions, shown by the green rectangle in Fig. 3. These 2-D marginals are extreme cases of non-Gaussian behaviour in the obtained posteriors. Note, however, that for target 1 (smaller perturbations), the mean and samples of the entire marginal distribution are closer to the target model compared to those for target 2 (larger perturbations).

tuning strategy. Empirically, we find that this strategy accelerates convergence significantly.

In the case of high data variance $\left(\sigma^{2}=10 \mu \mathrm{m}^{2}\right)$, the Markov chain converges relatively fast. Decorrelation length for many parameters is only 3 to 5 samples, after which on average the sampler has generated an independent sample. Satisfactory convergence of 1-D and 2-D marginals is achieved using 10'000 samples, though more samples would certainly be needed for higher-dimensional marginals or the full posterior. Re-tuning the mass matrix, as described above, allows for the chain with $\sigma^{2}=1 \mu \mathrm{m}^{2}$ to converge in approximately 3 times as many samples as in the $\sigma^{2}=10 \mu \mathrm{m}^{2}$ case. Although the chain with $\sigma^{2}=0.1 \mu \mathrm{m}^{2}$ is not run until means and variances appear stable, the convergence seems to be equally enhanced by re-tuning the mass matrix.

\subsection{Analysis of the posterior}

The means and standard deviations for the converged chains are shown in Figs. 9 and 11, respectively. As expected, the means differ strongly between the cases of high and medium

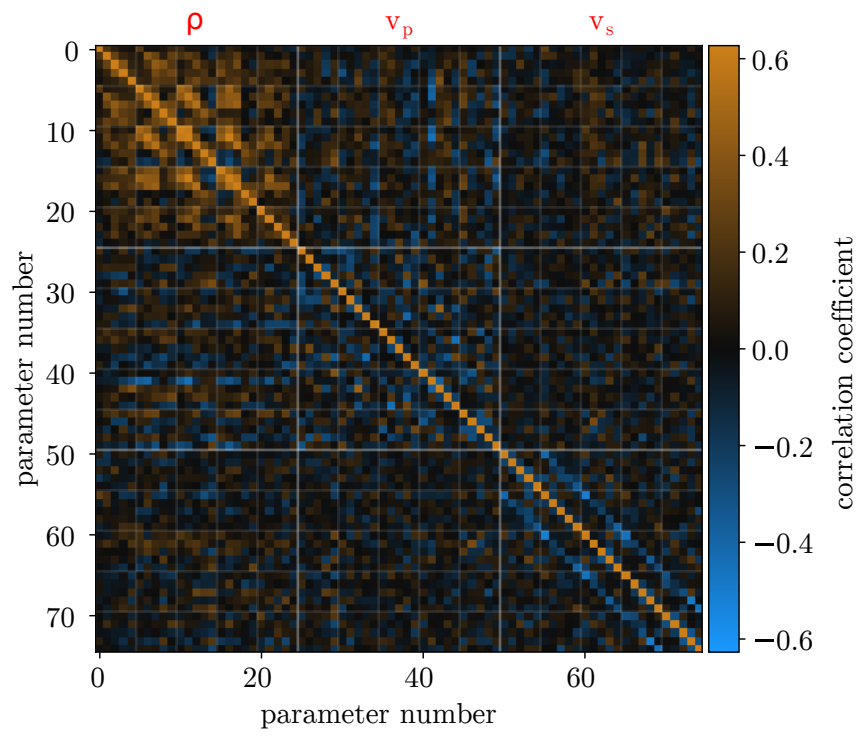

Figure 6: Correlation matrix for checkerboard target 2 shown in Fig. 1. Parameter correlations are significant within all parameter groups, but also occur between parameter groups. Density is positively correlated to surrounding densities, while velocities are negatively correlated to surrounding velocities.

data variance. Because the amount of effective samples differs per chain, some posterior quantities appear 'noisy' for the case of lower variance. This is effectively undersampling to a low degree.

While the means of $v_{s}$ only delineate the strongest discontinuities for $\sigma^{2}=10 \mu \mathrm{m}^{2}$, they provide a remarkably accurate image of the target model for the medium data variance of $\sigma^{2}=1$ $\mu \mathrm{m}^{2}$. The posterior mean values of $\rho$ resemble the target density mostly near discontinuities, which is plausible given that seismic waveforms are primarily sensitive to density gradients. In contrast to $v_{s}$ and $\rho$, the posterior mean of $v_{p}$ is hardly similar to the target model. This is partly due to the pronounced nonGaussianity of the $v_{p}$ posterior, exemplified by the 2-D posterior marginals shown in Fig. 10.

The standard deviations show again the strong influence of the data variance $\sigma^{2}$. P-wave velocity shows relatively low standard 

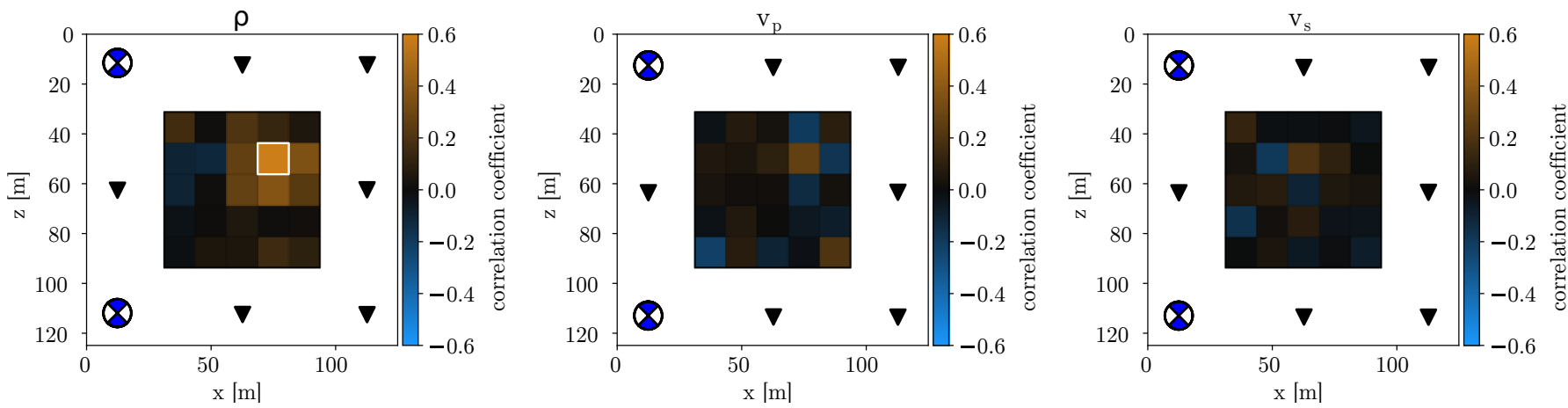

Figure 7: Correlations to parameter 8 for checkerboard target 2 shown in Fig. 1. Parameter 8 is highlighted by the white box. The positive correlation between densities is focused around parameter 8 , as seen in the left plot. Thus, if one of these densities is found to be higher (lower), neighboring densities are likely to be higher (lower). This is opposed to the (weak) negative correlation to some of the surrounding P-wave velocities as seen in the middle plot. If the density is found to be higher (lower), the surrounding velocities are expected to be lower (higher).

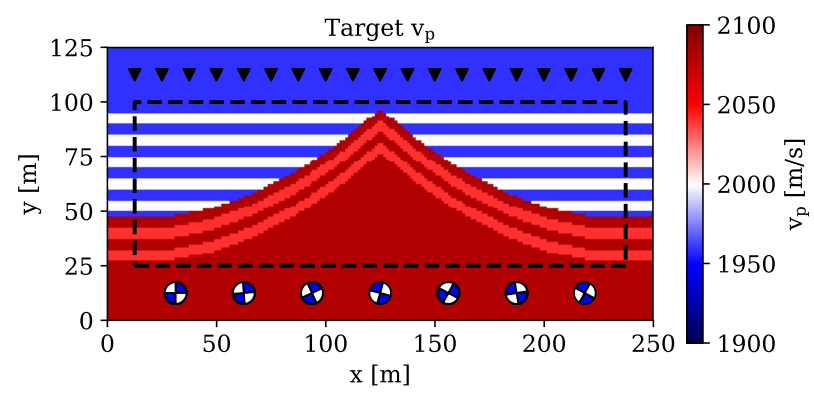

deviation along the direct wave paths. As expected, standard deviations of density are lower at discontinuities, and standard deviations for $v_{s}$ are lowest in regions of elevated $v_{s}$. For all parameters, posterior standard deviations are strongly model dependent, again highlighting the non-linear nature of the inverse problem. Changing assumed data noise, i.e., the data variance $\sigma^{2}$, not only modifies the magnitude of the posterior variance, but also its spatial distribution. The white regions of standard deviation in Figure 11 indicate parameters on which the standard deviation was not decreased with respect to the prior, ie. no knowledge was gained from the FWI experiment.

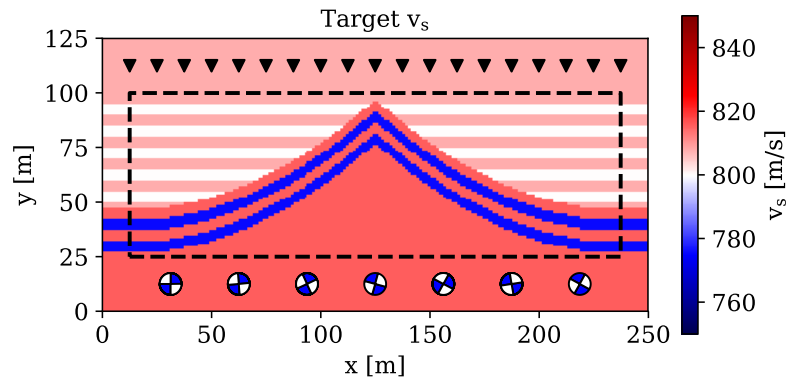

\section{Discussion}

In the following paragraphs we discuss further details of our method, including the analysis and acceleration of MCMC convergence, the recovery of density structure, and the future extension to real-data applications in 3-D.

\subsection{Convergence diagnostics}

While a large number of convergence diagnostics for MCMC methods have been developed $[75,76]$, none of these is universally applicable or useful [77]. Thus, convergence is relative to the information that one wishes to extract from the prior, or to the decisions one needs to make on its basis. Here we subjectively chose to assess convergence by monitoring means and covariances, as well as 1-D and 2-D marginals. This may be replaced by other metrics, depending on the application.

Furthermore, we ran chains from different initial models in order to detect trapping in a local minimum. This was the case, for instance, in an enlarged checkerboard inversion with $10 \times 10$ basis functions, corresponding to 300 model parameters (see chain $\mathrm{C}$ in Table 1).

Figure 8: Structural target with $10^{\prime} 800$ free parameters for $v_{p}$, $v_{s}$ and $\rho$. The dashed black line indicates the region within which parameters are allowed to vary. Sources and receivers are indicated by beachballs and black $\mathbf{\nabla}$ symbols, respectively.

\subsection{Parallel tempering}

A sampling approach that tries to mitigate local minima is parallel tempering or replica exchange, where the states of two Markov chains at different temperatures are randomly swapped $[78,72]$. Since one of the chains has a higher temperature, it has a higher probability of escaping local minima. 

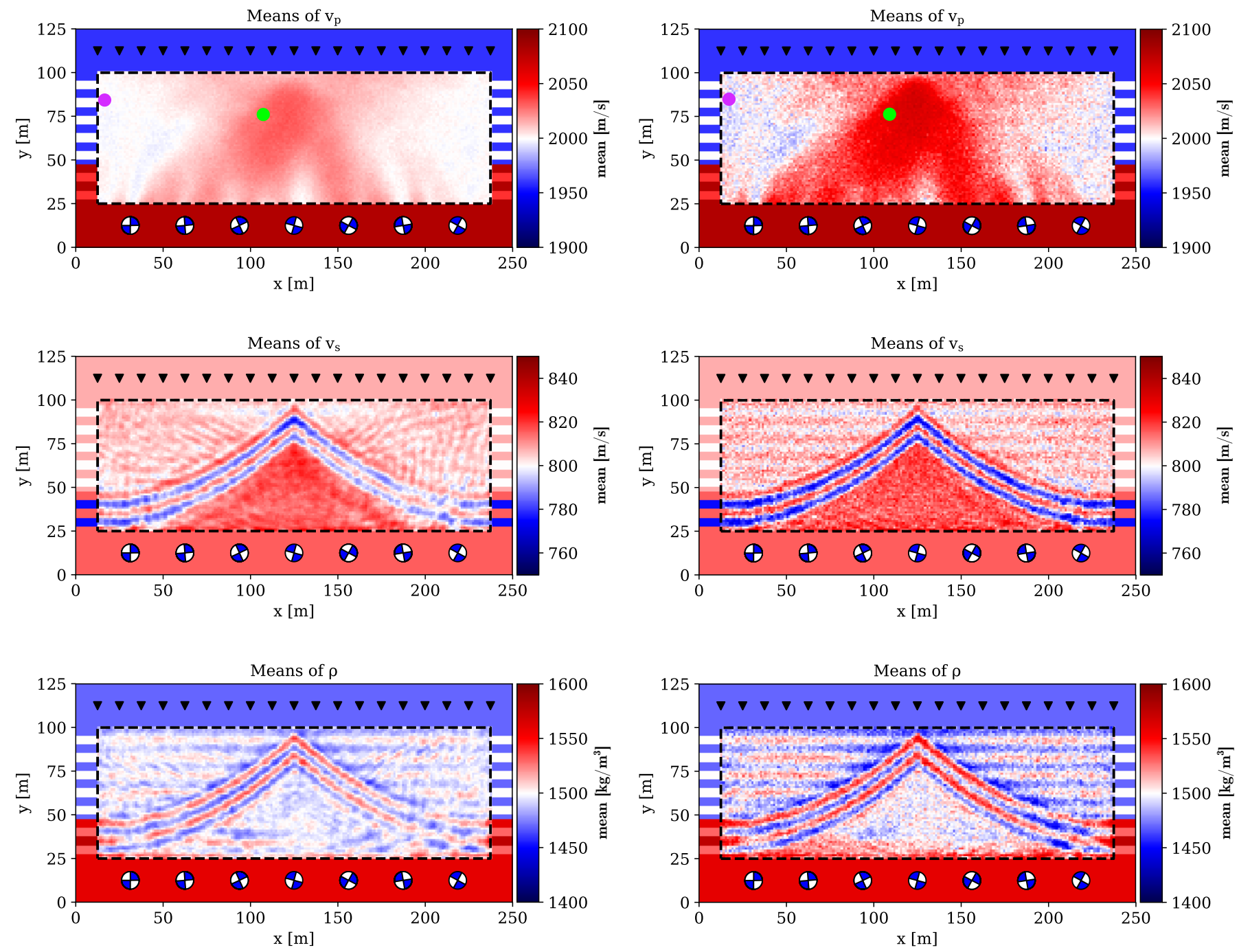

Figure 9: Posterior means of chains with $\sigma^{2}=10 \mu \mathrm{m}^{2}$ (left) and $\sigma^{2}=1 \mu \mathrm{m}^{2}$ (right). The means for the lower data variance $\left(\sigma^{2}=1 \mu \mathrm{m}^{2}\right)$ show a closer resemblance to the target model. However, they appear less smooth due to a stronger dependence of samples (undersampling). The $v_{p}$ mean is hardly similar to the $v_{p}$ target because the posterior is strongly non-Gaussian, as illustrated in Fig. 10. The green and purple dot respectively indicate parameter 8000 and 9720, the parameters visualized in Fig. 10. 

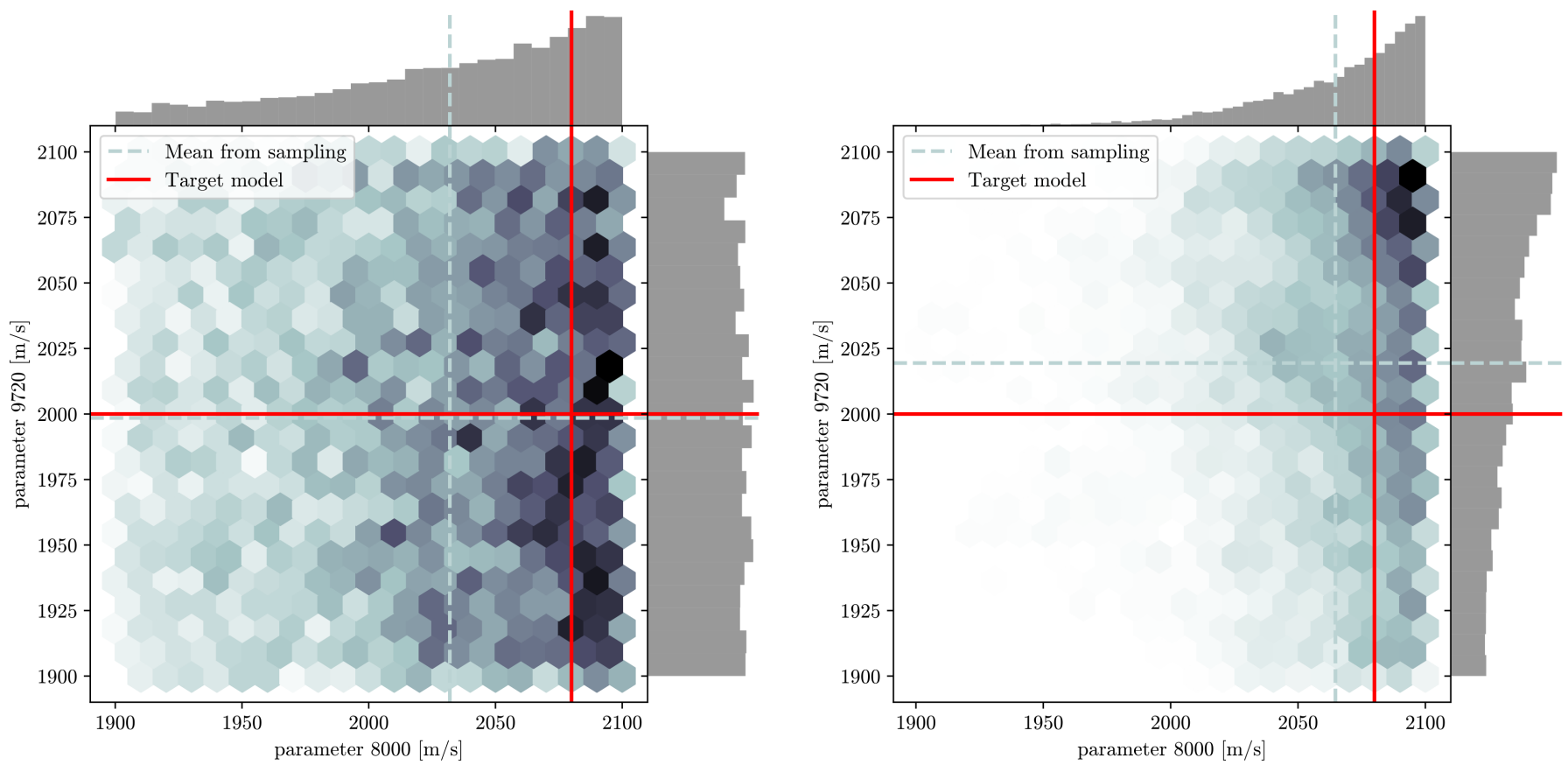

Figure 10: Examples of 2-D posterior marginal distributions for two neighboring $v_{p}$ parameters. Locations are indicated in Fig. 9. The prior is represented by the limits of the axes. The posterior marginals appear truncated by the prior, adding to the non-Gaussianity of the distribution. As expected, the marginal for $\sigma^{2}=1 \mu \mathrm{m}^{2}$ (right) is more localized than for $\sigma^{2}=10 \mu \mathrm{m}^{2}$ (left). The data is especially non-informative for parameter 9720 on the $\sigma^{2}=10 \mu \mathrm{m}^{2}$ (left) plot, where the posterior marginal strongly resembles the prior.

As a proof of concept, we implemented parallel tempering for the high-dimensional target shown in Fig. 8. We linked six Markov chains differing in temperature, i.e., data variance. The highest variance was at $\sigma^{2}=10 \mu \mathrm{m}^{2}$, and the spacing of the lower variances was chosen empirically, such that an acceptance rate between 20-80 \% was achieved. The result is chain $\mathrm{G}$ in Table 1. With six chains we were able to bridge variances from $\sigma^{2}=10 \mu \mathrm{m}^{2}$ to $\sigma^{2}=8.1 \mu \mathrm{m}^{2}$ using a logarithmic temperature spacing. On extrapolation, this would mean approximately 50 60 chains for an order of magnitude decrease in data variance. We expect parallel tempering to also benefit convergence of slowly mixing chains like chains $\mathrm{H}$ and $\mathrm{I}$.

\subsection{Tuning and the mass matrix}

The tuning of the mass matrix largely controls the efficiency of HMC. The mass matrix determines the relative speed in the different coordinate directions along a Hamiltonian trajectory, and it may be used for preferential sampling, e.g., of particularly smooth or rough models [67].

In this work, we applied an intuitive tuning where the diagonal elements of the mass matrix are adjusted using either a visual analysis of trace plots or a rough approximation of the variances using a small number of samples. Ideally, the mass matrix should adapt dynamically to the local curvature of the posterior, e.g., using second-derivative information from the local Hessian [79, 80, 81]. The testing of such Hessian-aware algorithms in the context of FWI is work in progress.

\subsection{Recovery of density structure}

A particularly noteworthy result of the HMC-based FWI is the recovery of a probabilistic density model that does not suffer from artificial biases introduced by regularization, needed to stabilize inversions for density using gradient-driven optimization [82, 18,74]. MCMC sampling allows density to vary regularization-free, as required by the data. This, in turn, avoids biases in seismic velocity variations that are often scaled a priori to density variations using empirical, but not universally valid, $v_{p, s} / \rho$ ratios [83].

\subsection{Towards real-data applications}

This work constitutes a pilot study, intended to establish a 2-D proof of concept for probabilistic full-waveform inversion based on Hamiltonian Monte Carlo sampling. The transition to 3-D real-data applications will require several improvements and additions to the current method.

Most importantly, the numerical modelling must be extended to 3-D and optimized for modern high-performance computers. GPU-enabled wave propagation codes are available for this purpose [84, 85, 86, 87, 88].

In this synthetic study, we have deliberately chosen a simple misfit functional, i.e., the $L_{2}$ waveform misfit (Eq. 14). In practice, this would be replaced by other measures of waveform similarity that are more robust and potentially introduce less trade-offs between seismic source parameters and Earth structure [29, 30, 31, 89, 32, 33, 34]. Furthermore, the observational error statistics associated with a specific misfit will need to be 

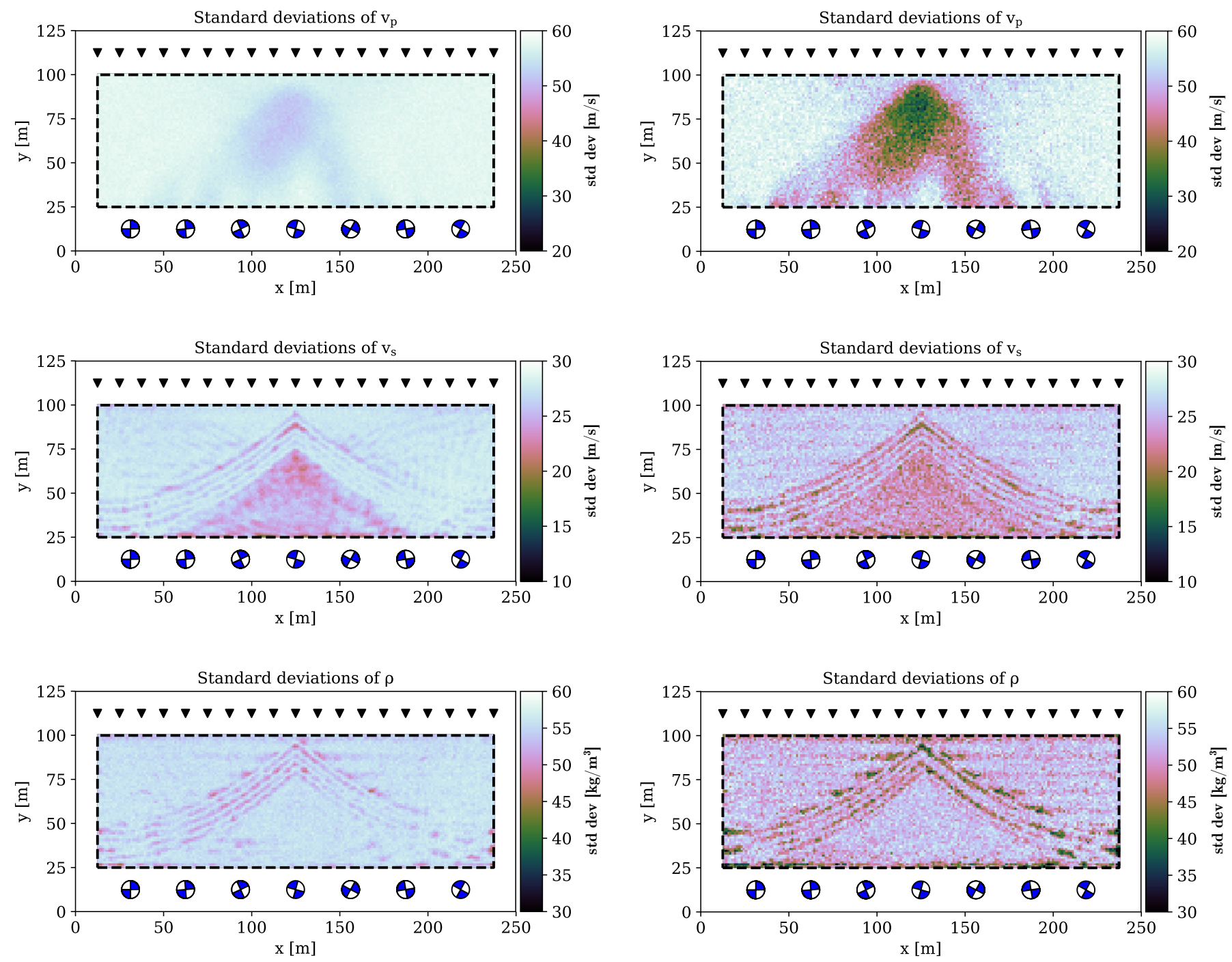

Figure 11: Posterior standard deviations for $\sigma^{2}=10 \mu \mathrm{m}^{2}$ (left) and $\sigma^{2}=1 \mu \mathrm{m}^{2}$ (right). Not only the magnitude but also the spatial distribution of posterior standard deviations depends strongly on $\sigma$. Density clearly has smaller standard deviation at discontinuities, whereas $v_{s}$ standard deviation is strongly dependent on the $v_{s}$ target itself. The maximum of the colorbars represent the prior standard deviations, thus white regions indicate parameters where no information was gained by the FWI experiment. 
analyzed carefully to ensure that the model space posterior is meaningful.

\section{Conclusions}

We have provided a proof of concept for a Bayesian elastic fullwaveform inversion in 2-D. This was intended to establish the methodological and computational basis for future extensions to real-data applications.

Key ingredients of our method are (1) a Hamiltonian Monte Carlo sampler that explores the full posterior distribution, (2) the computation of misfit derivatives with the help of adjoint techniques, and (3) a tuning strategy that adjusts the diagonal elements of the mass matrix to accounts for the different sensitivities of seismic velocities and density.

The method successfully works for two synthetic end-member scenarios with 75 and 32'400 dimensions, respectively. In both cases, the algorithm recovers important aspects of the posterior, which can be significantly non-Gaussian. In addition to $\mathrm{P}$-wave and $\mathrm{S}$-wave velocity, the sampling provides constraints on density structure that are free from subjective regularization artifacts, yielding the prior when the data itself is uninformative on a parameter

The most important conclusion is that further improvements listed in section 5.5 seem feasible, while certainly not being trivial. This suggests that $3-\mathrm{D}$ probabilistic FWI is within reach

\section{AcKNOWLEDGEMENTS}

We gratefully acknowledge fruitful discussions with our colleagues Andrew Curtis, Alison Malcolm, Klaus Mosegaard, Julien Thurin, Jean Virieux and Andrea Zunino. This work was supported by the Swiss National Supercomputing Center (CSCS) in the form of computing time grants c13 and s868.

\section{REFERENCES}

[1] A. Bamberger, G. Chavent, and P. Lailly. Une application de la théorie du contrôle à un problème inverse sismique. Ann. Geophys., 33:183-200, 1977.

[2] A. Bamberger, G. Chavent, Ch. Hemons, and P. Lailly. Inversion of normal incidence seismograms. Geophysics, 47:757-770, 1982.

[3] P. Lailly. The seismic inverse problem as a sequence of before stack migrations. In J.B. Bednar, R. Redner, E. Robinson, and A. Weglein, editors, Conference on Inverse Scattering: Theory and Application. Soc. Industr. appl. Math., Philadelphia, PA., 1983.

[4] A. Tarantola. Inversion of seismic reflection data in the acoustic approximation. Geophysics, 49:1259-1266, 1984.

[5] O. Gauthier, J. Virieux, and A. Tarantola. Two-dimensional nonlinear inversion of seismic waveforms: numerical results. Geophysics, 51:1387-1403, 1986.

[6] A. M. Dziewoński, B. H. Hager, and R. J. O’Connell. Large-scale heterogeneities in the lower mantle. J. Geophys. Res., 82:239-255, 1977.
[7] K. Aki, A. Christoffersson, and E. S. Husebye. Determination of three-dimensional seismic structure of the lithosphere. J. Geophys. Res., 81:277-296, 1976.

[8] W. Spakman, S. van der Lee, and R. van der Hilst. Traveltime tomography of the European-Mediterranean mantle down to 1400 km. Phys. Earth Planet. Int., 79:3-74, 1993.

[9] R.D. van der Hilst, S. Widiyantoro, and E.R. Engdahl. Evidence for deep mantle circulation grom global tomography. Nature, 386:578-584, 1997.

[10] S. Grand, R. VanDerHilst, and S. Widiyantoro. Global seismic tomography: A snapshot of convection in the earth. Geol. Soc. Am. Today, 7, No.4:1-7, 1997.

[11] A. Gorbatov and B. L. N. Kennett. Joint bulk-sound and shear tomography for Western Pacific subduction zones. Earth Planet. Sci. Lett., 210:527-543, 2003.

[12] K. Yomogida. Fresnel zone inversion for lateral heterogeneities in the Earth. Pure Appl. Geophys., 138:391-406, 1992.

[13] F.A. Dahlen, S.-H. Hung, and G. Nolet. Fréchet kernels for finite-frequency traveltimes - I. Theory. Geophys. J. Int., 141:157-174, 2000.

[14] W. Friederich. The S-velocity structure of the East Asian mantle from inversion of shear and surface waveforms. Geophys. J. Int., 153:88-102, 2003.

[15] K. Yoshizawa and B. L. N. Kennett. Multi-mode surface wave tomography for the Australian region using a 3-stage approach incorporating finite-frequency effects. J. Geophys. Res., 109:doi:10.1029/2002JB002254, 2004.

[16] K. Sigloch, N. McQuarrie, and G. Nolet. Twostage subduction history under north america inferred from multiple-frequency tomography. Nat. Geosc., 1: doi:10.1038/ngeo231, 2008.

[17] L. Sirgue, O. I. Barkved, J. Dellinger, J. Etgen, U. Albertin, and J. H. Kommedal. Full-waveform inversion: the next leap forward in imaging at Valhall. First Break, 28:65-70, 2010.

[18] V. Prieux, R. Brossier, S. Operto, and J. Virieux. Multiparameter full waveform inversion of multicomponent ocean-bottom-cable data from the Valhall field. Part 1: Imaging compressional wave speed, density and attenuation. Geophys. J. Int., 194:1640-1664, 2013.

[19] M. Warner, A. Ratcliffe, T. Nangoo, J. Morgan, A. Umpleby, N. Shah, V. Vinje, I. Stekl, L. Guasch, C. Win, G. Conroy, and A. Betrand. Anisotropic 3D full-waveform inversion. Geophysics, 78:R59-R80, 2013.

[20] P. Chen, L. Zhao, and T. H. Jordan. Full 3D tomography for the crustal structure of the Los Angeles region. Bull. Seismol. Soc. Am., 97:1094-1120, 2007.

[21] A. Fichtner, B. L. N. Kennett, H. Igel, and H.-P. Bunge. Full seismic waveform tomography for upper-mantle structure in the Australasian region using adjoint methods. Geophys. J. Int., 179:1703-1725, 2009.

[22] C. Tape, Q. Liu, A. Maggi, and J. Tromp. Seismic tomography of the southern California crust based upon spectral-element and adjoint methods. Geophys. J. Int., 180:433-462, 2010. 
[23] L. Krischer, H. Igel, and A. Fichtner. Automated largescale full seismic waveform inversion for North America and the North Atlantic. J. Geophys. Res., 123:doi: 10.1029/2017JB015289, 2018.

[24] S. W. French and B. A. Romanowicz. Whole-mantle radially anisotropic shear velocity structure from spectralelement waveform tomography. Geophys. J. Int., 199: 1303-1327, 2014.

[25] E. Bozdağ, D. Peter, M. Lefebvre, D. Komatitsch, J. Tromp, J. Hill, N. Podhorszki, and D. Pugmire. Global adjoint tomography: First-generation model. Geophys. J. Int., 207: 1739-1766, 2016.

[26] A. Fichtner, D.-P. van Herwaarden, M. Afanasiev, S. Simute, L. Krischer, Y. Cubuk-Sabuncu, T. Taymaz, L. Colli, E. Saygin, A. Villasenor, J. Trampert, P. Cupillard, H.-P. Bunge, and H. Igel. The Collaborative Seismic Earth Model: Generation I. Geophys. Res. Lett., 45:40074016, 2018.

[27] C. Bunks, F. M. Saleck, S. Zaleski, and G. Chavent. Multiscale seismic waveform inversion. Geophysics, 60:14571473, 1995.

[28] L. Sirgue and R. G. Pratt. Efficient waveform inversion and imaging: A strategy for selecting temporal frequencies. Geophysics, 69:231-248, 2004.

[29] Y. Luo and G. T. Schuster. Wave-equation traveltime inversion. Geophysics, 56:645-653, 1991.

[30] L. S. Gee and T. H. Jordan. Generalized seismological data functionals. Geophys. J. Int., 111:363-390, 1992.

[31] A. Fichtner, B. L. N. Kennett, H. Igel, and H.-P. Bunge. Theoretical background for continental- and global-scale full-waveform inversion in the time-frequency domain. Geophys. J. Int., 175:665-685, 2008.

[32] T. van Leeuwen and W. A. Mulder. A correlation-based misfit criterion for wave-equation traveltime tomography. Geophys. J. Int., 182:1383-1394, 2010.

[33] E. Bozdağ, J. Trampert, and J. Tromp. Misfit functions for full waveform inversion based on instantaneous phase and envelope measurements. Geophys. J. Int., 185:845-870, 2011.

[34] L. Métivier, R. Brossier, Q. Mérigot, E. Oudet, and J. Virieux. Measuring the misfit between seismograms using an optimal transport distance: application to full waveform inversion. Geophys. J. Int., 205:345-377, 2016.

[35] K. Mosegaard and A. Tarantola. Monte Carlo sampling of solutions to inverse problems. J. Geophys. Res., 100: 12431-12447, 1995.

[36] M. S. Sambridge and K. Mosegaard. Monte Carlo methods in geophysical inverse problems. Rev. Geophys., 40:doi: 10.1029/2000RG000089, 2002.

[37] P. Käufl, A. Fichtner, and H. Igel. Probabilistic full waveform inversion based on tectonic regionalisation - Development and application to the Australian upper mantle. Geophys. J. Int., 193:437-451, 2013.

[38] M. V. Afanasiev, R. G. Pratt, R. Kamei, and G. McDowell. Waveform-based simulated annealing of crosshole transmission data: A semi-global method for estimating seismic anisotropy. Geophys. J. Int., 199:1586-1607, 2014.
[39] M. Kotsi, A. E. Malcolm, and G. Ely. 4D full-waveform Metropolis-Hastings inversion using a local acoustic solver. SEG Expanded Abstracts, August 2018:doi:segam20182997858.1, 2018.

[40] A. Fichtner and J. Trampert. Resolution analysis in full waveform inversion. Geophys. J. Int., 187:1604-1624, 2011.

[41] T. Bui-Thanh, O. Ghattas, J. Martin, and G. Stadler. A computational framework for infinite-dimensional Bayesian inverse problems part I: The linearized case, with application to global seismic inversion. SIAM J. Sci. Comp., 35: A2494-A2523, 2013.

[42] A. Fichtner and T. van Leeuwen. Resolution analysis by random probing. J. Geophys. Res., 120: doi:10.1002/2015JB012106, 2015.

[43] L. Liu, D. Peter, and C. Tape. Square-root variable metric based elastic full-waveform inversion - Part 1: theory and validation. Geophys. J. Int., 218:doi:10.1093/gji/ggz188, 2019.

[44] L. Liu, D. Peter, and C. Tape. Square-root variable metric based elastic full-waveform inversion - Part 2: uncertainty estimation. Geophys. J. Int., 218:doi:10.1093/gji/ggz137, 2019.

[45] R. M. Neal. MCMC using Hamiltonian dynamics. In Handbook of Markov Chain Monte Carlo, page Chapter 5, 2011.

[46] M. Betancourt. A conceptual introduction to Hamiltonian Monte Carlo. arXiv:1701.02434 [stat.ME], 2017.

[47] M. K. Sen and R. Biswas. Tansdimensional seismic inversion using the reversible jump Hamiltonian Monte Carlo algorithm. Geophysics, 82:R119-R134, 2017.

[48] A. Fichtner, A. Zunino, and L. Gebraad. Hamiltonian Monte Carlo solution of tomographic inverse problems. Geophys. J. Int., 216:doi:10.1093/gji/ggy496, 2018.

[49] A. Fichtner and S. Simute. Hamiltonian Monte Carlo inversion of seismic sources in complex media. J. Geophys. Res., 123:doi:10.1002/2017JB015249, 2018.

[50] S. Chib and E. Greenberg. Understanding the MetropolisHastings algorithm. American Statistician, pages 327-335, 1995.

[51] J.-L. Lions. Contrôle optimal de systèmes gouvernés par des équations aux dérivées partielles. Dunod GauthierVillars, 1968.

[52] A. Tarantola. Theoretical background for the inversion of seismic waveforms, including elasticity and attenuation. Pure Appl. Geophys., 128:365-399, 1988.

[53] Q. Liu and J. Tromp. Finite-frequency kernels based on adjoint methods. Bull. Seismol. Soc. Am., 96:2383-2397, 2006.

[54] A. Fichtner, H.-P. Bunge, and H. Igel. The adjoint method in seismology - I. Theory. Phys. Earth Planet. Inter., 157: 86-104, 2006.

[55] A. Fichtner, H.-P. Bunge, and H. Igel. The adjoint method in seismology - II. Applications: traveltimes and sensitivity functionals. Phys. Earth Planet. Inter., 157:105-123, 2006. 
[56] R.-E. Plessix. A review of the adjoint-state method for computing the gradient of a functional with geophysical applications. Geophys. J. Int., 167:495-503, 2006.

[57] J. Virieux and S. Operto. An overview of full waveform inversion in exploration geophysics. Geophysics, 74:WCC127-WCC152, 2009.

[58] A. Fichtner. Full Seismic Waveform Modelling and Inversion. Springer, Heidelberg., 2010.

[59] Q. Liu and Y. Gu. Seismic imaging: from classical to adjoint tomography. Tectonophysics, 566-567:31-66, 2012.

[60] E. T. Jaynes. Probability Theory - The Logic of Science. Cambridge University Press, Cambridge, UK, 2003.

[61] A. Tarantola. Inverse problem theory and methods for model parameter estimation, 2nd edition. Society for Industrial and Applied Mathematics, Philadephia, 2005.

[62] N. Metropolis, A. W. Rosenbluth, M. N. Rosenbluth, A. H Teller, and E. Teller. Equations of state calculations by fast computing machines. J. Chem. Phys., 21:1087-1092, 1953.

[63] W. K. Hastings. Monte Carlo sampling methods using Markov Chains and their applications. Biometrika, 57: 97-109, 1970.

[64] S. Duane, A. D. Kennedy, B. J. Pendleton, and D. Roweth Hybrid Monte Carlo. Phys. Lett. B, 195:216-222, 1987.

[65] Michael Creutz. Global monte carlo algorithms for manyfermion systems. Physical Review D, 38(4):1228, 1988.

[66] L. D. Landau and E. M. Lifshitz. Course of Theoretical Physics, Volume 1, Mechanics, 3rd edition. Elsevier Butterworth Heinemann, Amsterdam, 1976.

[67] A. Fichtner and A. Zunino. Hamiltonian nullspace shuttles. Geophys. Res. Lett., 46:doi:10.1029/2018GL080931, 2019.

[68] J. Virieux. P-SV wave propagation in heterogeneous media: velocity-stress finite difference method. Geophysics, 51: 889-901, 1986.

[69] T. Bodin, M. Sambridge, N. Rawlinson, and P. Arroucau. Transdimensional tomography with unknown data noise. Geophys. J. Int., 189:1536-1556, 2012.

[70] J. A. Scales and R. Snieder. To Bayes or not to Bayes. Geophysics, 62:1045-1046, 1997.

[71] C. J. Geyer and E. A. Thompson. Annealing Markov Chain Monte Carlo with applications to ancestral inference. $J$. Am. Stat. Assoc., 90:909-920, 1995.

[72] M. S. Sambridge. A parallel tempering algorithm for probabilistic sampling and multi-modal optimization. Geophys. J. Int., 196:357-374, 2014.

[73] H. Igel, H. Djikpesse, and A. Tarantola. Waveform inversion of marine reflection seismograms for $\mathrm{P}$ impedance and Poisson's ratio. Geophys. J. Int., 124:363-371, 1996.

[74] N. Blom, C. Boehm, and A. Fichtner. Synthetic inversions for density using seismic and gravity data. Geophys. J. Int., 209:1204-1220, 2017.

[75] A. Gelman and D. B. Rubin. Inference from iterative simulation using multiple sequences. Stat. Sci., 7:457-511, 1992.
[76] A. E. Raftery and S. Lewis. How many iterations in the Gibbs sampler? Bayesian Stat., 4:763-773, 1992.

[77] M. K. Cowles. Markov chain Monte Carlo convergence diagnostics: A comparative review. J. Am. Stat. Ass., 91: 883-904, 1996.

[78] C. J. Geyer. Markov chain Monte Carlo maximum likelihood. In Comp. Sci. Stat., Proc. 23rd Symposium on the Interface. Interface Foundation of North America, 1991.

[79] Y. Qi and T. P. Minka. Hessian-based Markov chain Monte Carlo algorithms. In First Cape Cod Workshop on Monte Carlo Methods. 2002. URL https://www. microsoft.com/en-us/research/publication/ hessian-based-markov-chain-monte-carlo-algorithms/.

[80] J. Dahlin, F. Lindsten, and T. B. Schön. Quasi-Newton particle Metropolis-Hastings. IFAC-PapersOnLine, 48-28: 981-986, 2015.

[81] T. Fu, L. Luo, and Z. Zhang. Quasi-Newton Hamiltonian Monte Carlo. In Proc. 32nd Conf. Uncert. Art. Int., pages 212-221. AUAI Press, Arlington, U.S., 2016.

[82] D. Köhn, A. Kurzmann, A. Przebindowska, D. de Nil, and T. Bohlen. 2D elastic full waveform tomography of synthetic marine reflection seismic data. DGG Mitteilungen, 1/2010:23-27, 2010 .

[83] T. M. Brocher. Empirical relations between elastic wavespeeds and density in the Earth's crust. Bull. Seis. Soc. Am., 95(6):2081-2092, 2005.

[84] D. Komatitsch and J. Tromp. Spectral-element simulations of global seismic wave propagation, part II: 3-D models, oceans, rotation, and gravity. Geophys. J. Int., 150:303$318,2002$.

[85] D. Komatitsch and J. Tromp. Spectral-element simulations of global seismic wave propagation, part I: validation. Geophys. J. Int., 149:390-412, 2002.

[86] D. Peter, D. Komatitsch, Y. Luo, R. Martin, N. Le Goff, E. Casarotti, P. Le Loher, F. Magnoni, Q. Liu, C. Blitz, T. Nissen-Meyer, P. Basini, and J. Tromp. Forward and adjoint simulations of seismic wave propagation on fully unstructured hexahedral meshes. Geophys. J. Int., 186: 721-739, 2011.

[87] A. Gokhberg and A. Fichtner. Full-waveform inversion on heterogeneous HPC systems. Comp. Geosci., 89:260-268, 2016.

[88] M. V. Afanasiev, C. Boehm, M. van Driel, L. Krischer, M. Rietmann, D. A. May, M. G. Knepley, and A. Fichtner. Modular and flexible spectral-element waveform modelling in two and three dimensions. Geophys. J. Int., 216: doi: 10.1093/gji/ggy469, 2019.

[89] R. Brossier, S. Operto, and J. Virieux. Which data residual norm for robust elastic frequency-domain full waveform inversion? Geophysics, 75:R37-R46, 2010. 
Preprint - Bayesian elastic Full-Waveform Inversion using Hamiltonian Monte Carlo

\section{A Overview of Markov chains}

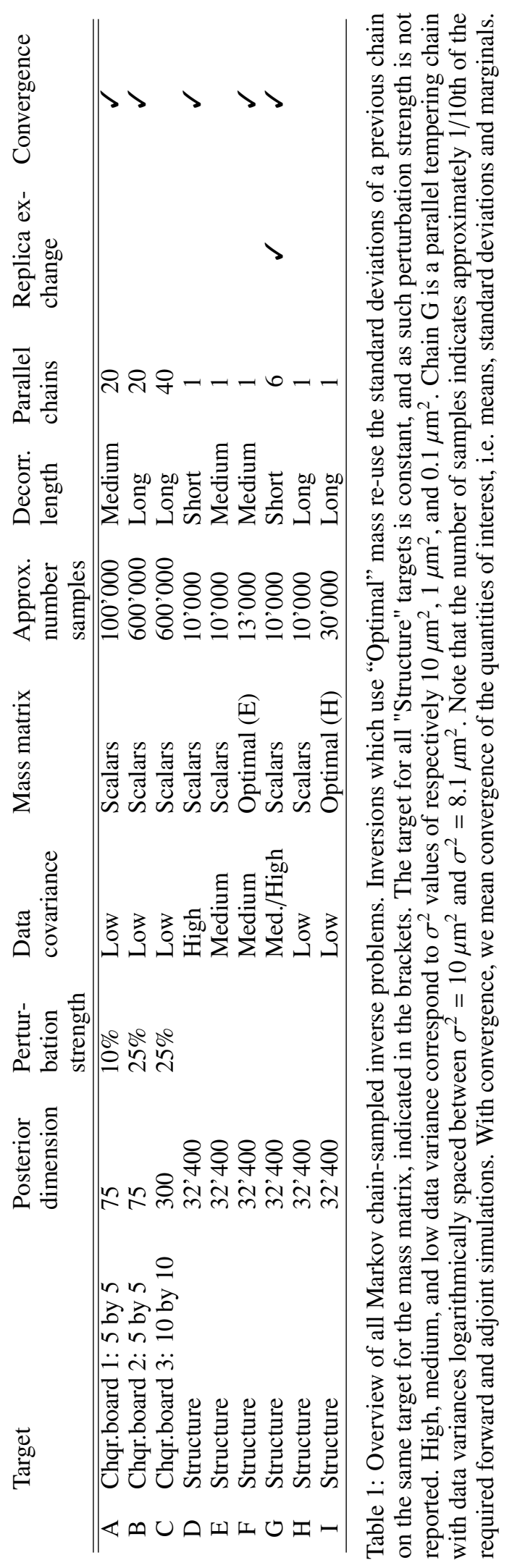

